IASSNS-HEP-93/7

March, 1993

\title{
ROLE OF SHORT DISTANCE BEHAVIOR IN OFF-SHELL OPEN-STRING FIELD THEORY
}

\author{
${\text { KEKE } \text { LI }^{\star} \text { AND EDWARD WitTEN }}^{\dagger}$ \\ School of Natural Sciences \\ Institute for Advanced Study \\ Olden Lane \\ Princeton, NJ 08540
}

\begin{abstract}
A recent proposal for a background independent open string field theory is studied in detail for a class of backgrounds that correspond to general quadratic boundary interactions on the world-sheet. A short-distance cut-off is introduced to formulate the theory with a finite number of local and potentially unrenormalizable boundary couplings. It is shown that renormalization of the boundary couplings makes both the world-sheet partition function and the string field action finite and cut-off independent, although the resulting string field action has an unpalatable dependence on the leading unrenormalizable coupling.
\end{abstract}

\footnotetext{
$\star$ W.M. Keck Foundation Fellow

$\dagger$ Research supported in part by NSF Grant PHY91-06210.
} 


\section{Introduction}

Recently, a new open string field action has been proposed using the BatalinVilkovisky formalism[1]. Formally, this action, defined in the space of all worldsheet open string theories, is background independent and gives the expected classical field equations and on-shell gauge symmetry.

It has been emphasized in [1] that the construction of the string field action has been formal because ultraviolet divergences associated with unrenormalizable local world-sheet interaction have been ignored. This question is crucial because the generic world-sheet theory is unrenormalizable; the massive states of the string are represented in the world-sheet Lagrangian by unrenormalizable interactions, as are high frequency modes of massless states. The difficulty in making sense of the generic two dimensional Lagrangian has indeed long been one of the main obstacles to progress in string theory; it severely limits applicability of the worldsheet approach to string theory.

Since it is hard to find a general way to remove the cut-off, one can simply define the string field action in a space of cut-off interactions. If the cut-off is strong enough, there appears to be no problem with any of the considerations of [1]. However, the expected classical solutions of the theory probably cannot be found in a space of world-sheet theories with strongly cut-off boundary interactions (since the standard perturbations are by dimension one local operators on the boundary which are not "soft"); and a space of such interactions probably cannot be defined in a background independent way (the obvious way to get a space of strongly cutoff theories is to start with a particular background, which cannot be "soft" since no theory is, and then add to it a family of soft perturbations). So it does not appear adequate to define the string field action only in a space of strongly cut-off interactions. However, one can begin by defining it in such a space and then try to remove the cut-off. In fact, that is what we will do in this paper.

The difficulties with unrenormalizable theories arise because the short distance behavior is out of control and depends on the specific Lagrangian; and therefore 
the world-sheet anomalies, which are so important in string theory, are also out of control. To probe these issues, we will consider a family of free field theories with quadratic but higher derivative boundary couplings; being free, these theories are tractable, but the short distance behavior depends on the specific couplings. For motivation, we first recall the example considered in [2]; the bulk action was the standard closed string action

$$
L_{\mathrm{I}}=\int_{\Sigma} \mathrm{d}^{2} \sigma \sqrt{h}\left(\frac{1}{8 \pi} h^{\alpha \beta} \partial_{\alpha} X_{\mu} \partial_{\beta} X_{\nu} \eta^{\mu \nu}+\frac{1}{2 \pi} b^{\alpha \beta} D_{\alpha} c_{\beta}\right)
$$

and the boundary action was

$$
L_{\mathrm{B}}^{\prime}=\oint_{\partial \Sigma} \mathrm{d} \theta\left(\frac{a}{2 \pi}+\sum_{\mu=1}^{26} \frac{u^{\mu}}{8 \pi} X_{\mu}^{2}\right)
$$

Here the world-sheet is a disc $\Sigma$ with unit radius, and $\left\{a, u^{\mu}\right\}$ is a set of parameters. The boundary interaction represents a quadratic tachyon background and is super-renomalizable; thus it only requires proper normal-ordering (corresponding to absorbing an infinite constant into $a$ ) to make both the world-sheet partition function and string field action well-defined.

The discussion in this paper will follow that of [2] closely, but now with the most general quadratic boundary action:

$$
L_{\mathrm{B}}=a+\frac{1}{8 \pi} \oint \mathrm{d} \theta \mathrm{d} \theta^{\prime} X_{\mu}(\theta) u^{\mu \nu}\left(\theta-\theta^{\prime}\right) X_{\nu}\left(\theta^{\prime}\right)
$$

At this stage we need to decide what kind of function $u$ is to be. The only evident notion of locality is that $u$ should be a finite sum of derivatives of delta functions:

$$
u^{\mu \nu}\left(\theta-\theta^{\prime}\right)=\sum_{r=0}^{s} t_{r}^{\mu \nu} \frac{d^{r}}{d \theta^{r}} \delta\left(\theta-\theta^{\prime}\right)
$$

with $t_{r}^{\mu \nu}$ being constants. With such a choice, most axioms of local quantum field theory are preserved (but world-sheet unitarity is lost because of the higher 
derivatives); the short distance behavior of the propagator depends on $s$, leading to some unpleasant properties that we will see later. Alternatively, one can try to take $s \rightarrow \infty$; this would even appear to be dictated by the fact that, once massive fields are excited in string theory, one should expect fields of arbitrarily high mass to be excited. But with $s=\infty$, the sum in (1.4) is no longer local. For instance, the "identity"

$$
X(\theta) X(\theta+\epsilon)=\sum_{n=0}^{\infty} \frac{\epsilon^{n}}{n !} X(\theta) \frac{d^{n}}{d \theta^{n}} X(\theta)
$$

shows that any bilocal expression can be expanded formally as an infinite sum of local operators. What kind of function we get upon taking $s \rightarrow \infty$ in (1.4) depends on what large $r$ behavior we assume for $t_{r}^{\mu \nu}$. For instance, a suitable condition on the $t_{r}^{\mu \nu}$ would give a class of strongly cut-off boundary interactions, as discussed above. One of the basic puzzles about our problem is that apart from the local interactions (finite $s$ ) which have their own difficulties that we have sketched, we do not know a natural class of boundary interactions to focus on.

The quadratic nature of the boundary interactions makes the world-sheet theory exactly soluble, even though the dependence of the short-distance behavior on the Lagrangian would usually be characteristic of unrenormalizable theories. This will be discussed in Section 2, where the exact partition function is determined from the exact Green's function. In practice, our way of grappling with the issues introduced above will be to introduce a regulator corresponding to a boundary cut-off $\epsilon$ that replaces $\delta\left(\theta-\theta^{\prime}\right)$ by $\delta_{\epsilon}\left(\theta-\theta^{\prime}\right)$ with $\lim _{\epsilon \rightarrow 0} \delta_{\epsilon}\left(\theta-\theta^{\prime}\right)=\delta\left(\theta-\theta^{\prime}\right)$. The regularized theory thus has a smooth coupling function $u^{\mu \nu}\left(\theta-\theta^{\prime}\right)$ and a non-local interaction (1.3). After computing the partition function and the string field action, we will then see to what extent it is possible to remove the cut-off. To do so, we will renormalize the local coupling parameters so that the partition function as a function of renormalized couplings remains finite as $\epsilon \rightarrow 0$. In fact, the quadratic nature of (1.3) implies that the only renormalization needed is to absorb into $a$ a divergent term, which is now a non-linear function of other couplings, rather than proportional to $u^{\mu}$ as in the case of (1.2). 
In Section 3, we proceed to analyse the string field action. The action is determined from world-sheet two-point functions of boundary interactions and their BRST transformation. Here we argue that to justify the formal considerations of [1], the BRST transformation of boundary operators should not be modified by the presence of boundary interactions; otherwise the proof that the antibracket is closed does not go through. This in fact is one reason that it is necessary to begin the construction in a space of cut-off boundary interactions; if the short distance behavior depends on the Lagrangian, the BRST transformation laws will also.

In trying to remove the cut-off, our main result is that the same renormalization that makes the partition function finite also makes the string field action finite. This is not obvious a priori. However, after we remove the cut-off, the fact that the short distance behavior of the local theory (1.3) depends on the boundary interaction comes back to haunt us in the following way. The string field action that we obtain is finite but has an explicit dependence on $s$ in the following sense: the action $S\left(t_{0}, \ldots, t_{s}\right)$ constructed with one value of $s$ does not coincide, if one sets $t_{s}=0$, with the action $S\left(t_{0}, \ldots, t_{s-1}\right)$ that one would obtain starting from the outset with a smaller value of $s$. This behavior is unpleasant, and we do not know the right interpretation.

\section{Partition Function}

The goal in this section is to solve the world-sheet matter theory defined by $L_{\mathrm{I}}+L_{\mathrm{B}}$ on the disc by determining its matter partition function. Since the action is quadratic, the partition function can simply be expressed as a determinant of a corresponding Gaussian kernel, which in this case is a differential operator on the circle, with a conventional (e.g. $\zeta$-function) regularization. The approach here will be different, in that the regulator will be introduced directly in the action by making $u\left(\theta-\theta^{\prime}\right)$ in (1.3) a smooth function for a non-zero cut-off $\epsilon$, and the local boundary interaction is recovered in the $\epsilon \rightarrow 0$ limit. The partition function will 
be determined by integrating a two-point Green's function, and will then be made finite (as $\epsilon \rightarrow 0$ ) by a renormalization counter-term.

Let the unit disc be parametrized by polar coordinates $(r, \theta)$ with the boundary at $r=1$, as well as by complex coordinates $(z, \bar{z})$ with $z=r \mathrm{e}^{i \theta}$. The variational principle applied to $(1.1)+(1.3)$ gives the following boundary condition for $X_{\mu}$ :

$$
\partial_{r} X_{\mu}(\theta)+\oint \mathrm{d} \theta^{\prime} u^{\mu \nu}\left(\theta-\theta^{\prime}\right) X_{\nu}\left(\theta^{\prime}\right)=0
$$

Here we have chosen $\eta^{\mu \nu}=\delta^{\mu \nu}$ so that the spacetime indices may be raised and lowered freely and later formulas may be simplified, but it is obvious how to restore the Minkowski metric $\eta^{\mu \nu}$ in what follows. The exact Green's function $G_{\mu \nu}(z, w)=$ $\left\langle X_{\mu}(z, \bar{z}) X_{\nu}(w, \bar{w})\right\rangle$ satisfying boundary condition (2.1) can be expressed as:

$$
\begin{aligned}
G_{\mu \nu}(z, w)= & -\delta_{\mu \nu}\left(\ln |z-w|^{2}+\ln |1-z \bar{w}|^{2}\right)+2\left(u_{0}^{-1}\right)_{\mu \nu} \\
& -\sum_{k=1}^{\infty}\left(\frac{2 u_{k}}{k\left(k+u_{k}\right)}(z \bar{w})^{k}+\frac{2 u_{-k}}{k\left(k+u_{-k}\right)}(\bar{z} w)^{k}\right)_{\mu \nu},
\end{aligned}
$$

where $u_{k}^{\mu \nu}=u_{-k}^{\nu \mu}=\oint \mathrm{d} \theta u^{\mu \nu}(\theta) \mathrm{e}^{-i k \theta}$ is the Fourier mode of coupling function. When both positions are on the boundary, with $z=\mathrm{e}^{i \theta}$ and $w=\mathrm{e}^{i \theta^{\prime}}$, the Green's function becomes:

$$
G_{\mu \nu}\left(\theta, \theta^{\prime}\right)=\sum_{k \in Z}\left(\frac{2}{|k|+u_{k}}\right)_{\mu \nu} \mathrm{e}^{i k\left(\theta-\theta^{\prime}\right)}
$$

which determines the partition function $Z$ through the differential equations:

$$
\frac{\partial}{\partial u_{k}^{\mu \nu}} \ln Z=-\frac{1}{16 \pi^{2}} \oint \mathrm{d} \theta \mathrm{d} \theta^{\prime} \mathrm{e}^{i k\left(\theta-\theta^{\prime}\right)}\left\langle X_{\mu}(\theta) X_{\nu}\left(\theta^{\prime}\right)\right\rangle=-\frac{1}{2}\left(\frac{1}{|k|+u_{-k}}\right)_{\mu \nu} .
$$

The partition function determined by (2.4) is valid for general (non-local) boundary couplings. Consider now boundary interactions involving a finite number 
of local couplings:

$$
L_{\mathrm{B}}=a+\frac{1}{8 \pi} \oint \mathrm{d} \theta \sum_{r=0}^{s} i^{r} t_{r}^{\mu \nu} X_{\mu} \partial_{\theta}^{r} X_{\nu}(\theta)
$$

This corresponds to $u^{\mu \nu}(\theta)=\sum_{r=0}^{s}(-i)^{r} t_{r}^{\mu \nu} \delta^{(r)}(\theta)$, or equivalently, $u_{k}^{\mu \nu}=$ $\sum_{r=0}^{s} t_{r}^{\mu \nu} k^{r}$. Now we introduce the short-distance cut-off $\epsilon$ by taking:

$$
u_{k}^{\mu \nu}=\sum_{r=0}^{s} t_{r}^{\mu \nu} k^{r} \mathrm{e}^{-|k| \epsilon}
$$

This is essentially a point-splitting regulator, and the particular form of the cutoff dependence is chosen here for later convenience. Viewed as a function of the coupling parameters $t_{r}^{\mu \nu}=(-1)^{r} t_{r}^{\nu \mu}$ and $a$, the partition function now satisfies

$$
\frac{\partial}{\partial t_{r}^{\mu \nu}} \ln Z_{s}=-\frac{\delta_{r, 0}}{2}\left(u_{0}^{-1}\right)_{\mu \nu}-\frac{1}{2} \sum_{k=1}^{\infty}\left(\left(\frac{k^{r} \mathrm{e}^{-k \epsilon}}{k+u_{k}}\right)_{\nu \mu}+(-1)^{r}\left(\frac{k^{r} \mathrm{e}^{-k \epsilon}}{k+u_{k}}\right)_{\mu \nu}\right),
$$

where the subscript $s$ indicates explicitly that the leading boundary coupling is $t_{s}$. Using also $\frac{\partial}{\partial a} \ln Z_{s}=-1$, we obtain

$$
Z_{s}=\left(\operatorname{det} t_{0}\right)^{-1 / 2} \cdot \mathrm{e}^{-a} \cdot \prod_{k=1}^{\infty} \operatorname{det}\left(1+k^{-1} u_{k}\right)^{-1 / 2}
$$

The partition function (2.8) is divergent as $\epsilon \rightarrow 0$. The divergence comes from the infinite sum in (2.7) for $r=s$ and $r=s-1$. To make sense of the partition function as $\epsilon \rightarrow 0$, one would like to view the "bare" couplings $t_{r}$ and $a$ as appropriate functions of "renormalized" couplings $t_{r}^{\prime}$ and $a^{\prime}$ and the cut-off $\epsilon$, such that the "renormalized" partition function, $\left.Z_{s}^{\prime}\left(t^{\prime}, a^{\prime} ; \epsilon\right) \equiv Z_{s}\left(t\left(t^{\prime}, a^{\prime}, \epsilon\right), a\left(t^{\prime}, a^{\prime}, \epsilon\right) ; \epsilon\right)\right)$, now viewed as a function of renormalized couplings, is finite in the limit $\epsilon \rightarrow 0$. It is easy to see that, for this quadratic theory, it is sufficient to take $t_{r}=t_{r}^{\prime}$ and $a=a\left(a^{\prime}, t_{r}^{\prime}, \epsilon\right)=a^{\prime}+\Delta a\left(t_{r}, \epsilon\right)$, and simply choose the counter-term $\Delta a$ to cancel 
the divergence in the partition function. To analyse this in detail, it is convenient to consider the generating function:

$$
\ln Z_{s}^{\prime}\left(t_{r}, a^{\prime} ; \epsilon\right)=-\frac{1}{2} \operatorname{Tr} \ln t_{0}-\operatorname{Tr} \sum_{k=1}^{\infty} \ln \left(1+k^{-1} u_{k}\right)-\Delta a-a^{\prime}
$$

where $u_{k}$ is given by (2.6). With cut-off $\epsilon \neq 0$, one may take derivatives with respect to $t_{r}$ of the infinite sum in (2.9), and show that the $\epsilon \rightarrow 0$ divergent term is not a constant but depends on $t_{s}$ and $t_{s-1}$. Furthermore, the dependence on $t_{s-1}$ is only linear. Thus in a "minimal subtraction" scheme the counter-term $\Delta a$ may be chosen to depend only on those two coupling parameters (for $s \geq 1$ ):

$$
\Delta a=-\operatorname{Tr} \sum_{k=1}^{\infty}\left(\ln \left(1+t_{s} k^{s-1} \mathrm{e}^{-k \epsilon}\right)+\frac{t_{s-1} k^{s-2} \mathrm{e}^{-k \epsilon}}{1+t_{s} k^{s-1} \mathrm{e}^{-k \epsilon}}\right) .
$$

Once the divergences are cancelled, one may take the $\epsilon \rightarrow 0$ limit of (2.9) or (2.8) to obtain the "renormalized" generating function or partition function. The generating function $\ln Z_{s}^{\prime}$ so determined is exact and generates arbitrary correlation functions of the (integrated) boundary operators associated with the couplings $t_{r}, 0 \leq r \leq s$. In particular all these correlation functions are finite once $\ln Z_{s}^{\prime}$ is made finite. The correlation functions of other composite boundary operators are not generated by $\ln Z_{s}^{\prime}$ and those of operators with higher dimensions, such as $X_{\mu} \partial_{\theta}^{r} X_{\nu}(\theta)$ for $r>s$, will still be divergent. (Correlation functions of bulk operators can be computed using Wick's theorem from the exact Green's function (2.2), and they have the usual short-distance behavior.)

It is worthwhile to consider special examples with $s=0,1$. The $s=0$ case corresponds to quadratic tachyon considered in [2]. One finds that the divergent part of the bare partition function is linear in $t_{0}$, and the minimal counter-term $\Delta a$ can be chosen to be

$$
\Delta a=-\operatorname{Tr}\left(t_{0}\right) \sum_{k=1}^{\infty} \frac{\mathrm{e}^{-k \epsilon}}{k}, \quad s=0
$$

This is the same logarithmically divergent counter-term as used in the normal- 
ordering prescription in [2]. With this counter-term, the renormalized partition function $Z_{0}^{\prime}$ can be seen to agree with that of [2].

For $s=1$, the second term in (2.10) is $\ln \epsilon$ divergent, while the first term contains an $\epsilon^{-1}$ divergence and a finite term. Defining $\Delta a$ without the finite term and using $t_{r}^{\mu \nu}=-(-1)^{r} t_{r}^{\nu \mu}$ to simplify further, one finds

$$
\Delta a=\epsilon^{-1} \sum_{m=0}^{\infty} \frac{\operatorname{Tr}\left(t_{1}^{2 m+2}\right)}{(2 m+2)^{2}}+\ln \epsilon \sum_{m=0}^{\infty} \frac{\operatorname{Tr}\left(t_{0} t_{1}^{2 m}\right)}{2 m+1}, \quad s=1 .
$$

This can also be compared with known results. Consider the case $t_{0}=0$, which describes open string coupled to a background $U(1)$ gauge field with constant field strength $t_{1}^{\mu \nu}$. There are $X_{\mu}$ zero modes and the partition function will be proportional to the spacetime volume, as indicated by the first factor in (2.8). The free energy (per unit volume) $\ln Z_{1}\left(t_{1}\right)$ is defined by $(2.9)$ without the first term. After substracting the counterm-term (2.12) and setting $a^{\prime}=0$ one finds

$$
\ln Z_{1}^{\prime}\left(t_{1}\right)=\frac{1}{4} \operatorname{Tr} \ln \left(1-t_{1}^{2}\right)
$$

in a complete agreement with earlier computations $[3,4]$. It is easy to see that the particular regulator and the minimal counter-term (2.12) used here is in fact equivalent to the $\zeta$-function regularization of [3].

Although the minimal subtraction scheme is most natural and reproduces earlier results for $s=0,1$ as remarked above, one may inquire whether other choices of $\Delta a$ with different finite part might be more natural for general $s$. Recall that in the theory of renormalization, ambiguities involving finite counter-terms are removed by renormalization conditions and possibly some other physical requirements such as symmetries. In the present problem, a natural requirement is the $s$ independence of the partition function in the sense that

$$
\left.Z_{s}^{\prime}\left(a^{\prime} ; t_{0}, \ldots, t_{s-1}, t_{s}\right)\right|_{t_{s}=0}=Z_{s-1}^{\prime}\left(a^{\prime} ; t_{0}, \ldots, t_{s-1}\right)
$$

as well as similar relations for the renormalized correlation functions in the $Z_{s}$ and $Z_{s-1}$ theories. This requirement is satisfied at $s=1$ by the minimal counter-terms 
(2.11) and (2.12), but is not satisfied by minimal counter-term (2.10) for $s>1$. In fact $\ln Z_{s}^{\prime}$ determined from (2.9) and (2.10) diverges as $t_{s} \rightarrow 0$. Such singular behavior of the renormalized theory as $t_{s} \rightarrow 0$ is not unexpected in general, since $t_{s}$ (for $s>1$ ) is the leading "unrenormalizable" coupling which dominates the short-distance behavior. Of course in a theory with a cut-off one may set $t_{s}=0$ without creating divergences. Then the failure of the partition function to satisfy (2.14) is due to the fact that the two limiting processes $\epsilon \rightarrow 0$ and $t_{s} \rightarrow 0$ do not commute.

The situation is simpler in the present case, as the bare partition function (2.8) is naively $s$-independent and thus the $s$-dependence of the renormalized partition function comes solely from the $s$-dependence of the minimal counter-term $\Delta a$. To satisfy (2.14) one simply needs to choose a non-minimal but $s$-independent counter-term $\Delta a$. There are many choices. The simplest one is to take $\Delta a=$ $-\operatorname{Tr} \sum_{k=1}^{\infty} \ln \left(1+k^{-1} u_{k}\right)$, and the renormalized partition function following from $(2.9)$ is

$$
Z_{s}=\left(\operatorname{det} t_{0}\right)^{-1 / 2} \cdot \mathrm{e}^{-a^{\prime}}
$$

independent of all couplings except $t_{0}$, corresponding to normally ordering $X \partial_{\theta}^{r} X$ to have zero expectation value. But this does not give the $s=0$ and $s=1$ results expected from minimal counter-terms and from earlier computations. There is a more complicated and but apparently natural choice that does give the expected $s=0$ and $s=1$ results. It gives the following $s$-independent renormalized generating function (with cut-off $\epsilon$ set to zero):

$\ln Z_{s}^{\prime}=-\frac{1}{4} \operatorname{Tr} \ln \frac{t_{0}^{2}}{1-t_{1}^{2}}+\operatorname{Tr} \sum_{k=1}^{\infty}\left(\ln \left(1-\frac{t_{0}}{k+\sum_{r=0}^{s} t_{r} k^{r}}\right)+\frac{t_{0}}{k+\sum_{r=1}^{s} t_{r} k^{r}}\right)+a^{\prime}$.

Note that since all (integrated) correlation functions of the quadratic boundary operators in the world-sheet action can be obtained by differentiation of the generating function $\ln Z_{s}^{\prime}$ with respect to the coulplings, they will also be $s$-independent. More generally, arbitrary correlation functions of interior or boundary operators 
may be obtained by using Wick's theorem from the exact Green's function (2.2), and they are manifestly $s$-independent as well.

\section{String Field Action}

Let the boundary action be represented by $\oint \mathrm{d} \theta b_{-1} O$, where $b$ is the antighost and $O$ has ghost number one. Introduce a basis $\left\{O_{i}\right\}$ of ghost number one boundary operators, so that $O=\sum_{i} x^{i} O_{i}$. Here the coupling constants $x_{i}$ may be viewed as parametrizing a point in the space of world-sheet theories and thus also as parametrizing a string field configuration. The string field action is defined in terms of world-sheet correlation functions by $[1,2]$ :

$$
\begin{aligned}
\mathrm{d} S & =\frac{1}{2} \sum_{i, j} x^{j} \mathrm{~d} x^{i} \oint \mathrm{d} \theta \mathrm{d} \theta^{\prime}\left\langle O_{i}(\theta)\left\{Q, O_{j}\right\}\left(\theta^{\prime}\right)\right\rangle \\
& =\frac{1}{2} \oint \mathrm{d} \theta \mathrm{d} \theta^{\prime}\left\langle\mathrm{d} O(\theta)\{Q, O\}\left(\theta^{\prime}\right)\right\rangle,
\end{aligned}
$$

where $\left\{Q, O_{j}\right\}$ is the BRST transformation of the boundary operator $O_{j}$.

Despite the boundary interaction, a conserved BRST current $\left(J_{z}, J_{\bar{z}}\right)$ exists in the interior, given by that of standard closed string theory. The action of BRST charge on a boundary operator $Q_{j}$ is

$$
\left\{Q, O_{j}\right\}=\oint_{C} \frac{\mathrm{d} z}{2 \pi i} J_{z}(z) O_{j}-\oint_{\bar{C}} \frac{\mathrm{d} \bar{z}}{2 \pi i} J_{\bar{z}}(\bar{z}) O_{j},
$$

where $C$ is a contour approaching the boundary of the disc and $\bar{C}$ is its image under $z \rightarrow 1 / z$. Unrenormalizable boundary couplings $\left(t_{r}\right.$ for $r>1$ ) in the absence of a cut-off modify the short-distance behavior of the exact boundary Green's function and thus the canonical structure between boundary operators, because these boundary interactions dominate the (interior) kinetic term in the worldsheet action. If the exact Green's function is used in extracting short-distance behavior in (3.2), the BRST transformation will cease to be geometrical, e.g., 
$\left\{Q, X_{\mu}(\theta)\right\} \neq c \partial_{\theta} X_{\mu}(\theta)$, where $c$ is the $\theta$ component of the ghost field on the boundary.

The BRST invariance of the antibracket defined on the space of world-sheet theories in fact requires that the BRST transformation law should be not be modified by the boundary interaction. This invariance is the statement that $\mathrm{d} S$ defined in (3.1) is indeed closed, and in proving this crucial property[1], one has made an important assumption that

$$
\frac{\partial}{\partial x^{j}}\left\{Q, O_{i}\right\}=0
$$

in notations described above. (In a more direct argument that $\mathrm{d} S$ is indeed locally exact [5], this condition must also be used.) Thus, to define the string field action, it appears that one must begin by working in a space of cut-off boundary interactions, for which (3.3) holds, and then if one wishes one can try to remove the cut-off.

For a general theory with quadratic interaction (1.3), we can take $O(\theta)$ to be of the form

$$
O(\theta)=c(\theta)\left(\frac{a}{2 \pi}+\frac{1}{8 \pi} X_{\mu}(\theta) \oint \mathrm{d} \theta^{\prime} u^{\mu \nu}\left(\theta-\theta^{\prime}\right) X_{\nu}\left(\theta^{\prime}\right)\right) \equiv c(\theta) V(\theta)
$$

The BRST transformation of $O(\theta)$ can be computed from (3.2) with the standard BRST current in closed string theory. In this computation, the matter stress energy tensor will be contracted with the matter part of (3.4) to extract short-distance singularities. There will be no double $\langle X X\rangle$ contractions here, since for a smooth (non-local) coupling function $u\left(\theta-\theta^{\prime}\right), X_{\mu}(\theta)$ and $X_{\nu}\left(\theta^{\prime}\right)$ in $O(\theta)$ are located at separate points on the boundary. For single contraction alone (and with the same short-distance behavior of two-point Green's function as in the free theory), one has the general formula $\left\{Q, X_{\mu}(\theta)\right\}=c \partial_{\theta} X_{\mu}(\theta)$. Applying this to (3.4), one finds:

$$
\{Q, O(\theta)\}=c c^{\prime} V(\theta)-\frac{1}{8 \pi} c X_{\mu}(\theta) \oint \mathrm{d} \theta^{\prime} u^{\mu \nu}\left(\theta-\theta^{\prime}\right) c\left(\theta^{\prime}\right) \partial_{\theta^{\prime}} X_{\nu}\left(\theta^{\prime}\right)
$$

Substituting (3.5) in (3.1) and evaluating the ghost correlation function according 
to:

$$
\left\langle c\left(\theta^{\prime \prime}\right) c(\theta) c\left(\theta^{\prime}\right)\right\rangle=2\left(\sin \left(\theta-\theta^{\prime}\right)+\sin \left(\theta^{\prime}-\theta^{\prime \prime}\right)+\sin \left(\theta^{\prime \prime}-\theta\right)\right)
$$

one obtains:

$$
\mathrm{d} S=\oint \mathrm{d} \theta \mathrm{d} \theta^{\prime}\left\langle\mathrm{d} V(\theta) \cdot\left(A\left(\theta^{\prime}\right)+\cos \left(\theta-\theta^{\prime}\right) B\left(\theta^{\prime}\right)+\sin \left(\theta-\theta^{\prime}\right) C\left(\theta^{\prime}\right)\right)\right\rangle
$$

where matter boundary operators $A, B$ and $C$ are given by

$$
\begin{aligned}
& A(\theta)=-V(\theta)+\frac{1}{8 \pi} X_{\mu}(\theta) \oint \mathrm{d} \theta^{\prime} \sin \left(\theta-\theta^{\prime}\right) u^{\mu \nu}\left(\theta-\theta^{\prime}\right) \partial X_{\nu}\left(\theta^{\prime}\right), \\
& B(\theta)=\frac{1}{4 \pi} X_{\mu}(\theta) \oint \mathrm{d} \theta^{\prime} u^{\mu \nu}\left(\theta-\theta^{\prime}\right) \partial X_{\nu}\left(\theta^{\prime}\right), \\
& C(\theta)=0 .
\end{aligned}
$$

Given the exact generating function $\ln Z$ for correlation functions of the boundary operators, it is straightforward to evaluate $\mathrm{d} S$ from (3.7). To simplify the discussion here, we shall use a general result proven in [2] to write down $S$ directly. This result states that if the boundary interaction $V(\theta)$ as introduced in (3.4) and the operator $A(\theta)$ as in (3.7) have the expansion in terms of a basis $\left\{V_{i}(\theta)\right\}$ of matter operators:

$$
V(\theta)=\sum_{i} x^{i} V_{i}(\theta), \quad A(\theta)=\sum_{i} \alpha^{i} V_{i}(\theta)
$$

then the action is given by

$$
S=\left(\sum_{i} \alpha^{i} \frac{\partial}{\partial x^{i}}+g\right) Z\left(x^{j}\right)
$$

where $Z$ is the world-sheet partition function and $g$ is a constant. Here the set of couplings is $\left\{x^{i}\right\}=\left\{a, u_{k}^{\mu \nu}\right\}$, with associated basis $\left\{V_{i}(\theta)\right\}=\left\{\partial_{x^{i}} V(\theta)\right\}$. A simple 
calculation following these definitions gives the corresponding functions $\left\{\alpha^{i}\right\} \equiv$ $\left\{\alpha, \alpha_{k}^{\mu \nu}\right\}:$

$$
\alpha=-a, \quad \alpha_{k}^{\mu \nu}=\frac{1}{2} k\left(u_{k+1}^{\mu \nu}-u_{k-1}^{\mu \nu}\right)-u_{k}^{\mu \nu}
$$

To determine the constant $g$, one may compute $S$ directly from (3.7) for special couplings. Such a computation with $u_{k}^{\mu \nu}=\delta^{\mu \nu} u_{k}$ yields $g=1$. This, together with (3.10) and (3.11), gives the string field action for general non-local quadratic boundary interaction:

$$
S=\left(\sum_{k \in Z}\left(\frac{1}{2} k\left(u_{k+1}^{\mu \nu}-u_{k-1}^{\mu \nu}\right)-u_{k}^{\mu \nu}\right) \frac{\partial}{\partial u_{k}^{\mu \nu}}+a+1\right) Z(u, a)
$$

where the partition function $Z$ is determined by (2.4).

Note that since (3.12) is derived for non-local boundary interactions, there is no ultraviolet divergence. In particular, both formula (3.10) and the BRST invariance $\mathrm{d}^{2} S=0$ (which must be used to derive (3.10)[2]) can be established rigorously. Now let the non-local coupling function $u\left(\theta-\theta^{\prime}\right)$ represent a set of local couplings $\left\{t_{r}, r=0, \ldots, s\right\}$ with boundary cut-off $\epsilon$ as introduced in Section 2. In Fourier modes, this is given by (2.6),

$$
u_{k}=\sum_{r=0}^{s} t_{r} k^{r} \mathrm{e}^{-|k| \epsilon}
$$

Applying this change of variables to (3.12), one finds explicit $\epsilon$-dependence, as well as implicit $\epsilon$-dependence through partition function $Z$. To remove the cut-off, one must show that the action as a function of renormalized coupling has a finite limit as $\epsilon \rightarrow 0$. Because of various sources of $\epsilon \rightarrow 0$ divergence in $S$, it appears at first almost impossible that the counter-term in $a=a^{\prime}+\Delta a$ used in Section 2 to make partition function finite could also make $S$ finite. But this turns out to be the case, as will be shown presently. 
After the change of variables (3.13), the action (3.12) becomes:

$$
\begin{aligned}
\frac{S_{s}}{Z_{s}} & =\frac{1}{2}\left(\mathrm{e}^{-\epsilon}+\mathrm{e}^{\epsilon}\right) \sum_{r=1}^{s} \sum_{m=0}^{\left[\frac{r-1}{2}\right]} c_{2 m+1}^{r} t_{r}^{\mu \nu} \frac{\partial \ln Z_{s}}{\partial t_{r-2 m}^{\mu \nu}}-\sum_{r=0}^{s} t_{r}^{\mu \nu} \frac{\partial \ln Z_{s}}{\partial t_{r}^{\mu \nu}}+a+1 \\
& +\left(\mathrm{e}^{-\epsilon}-\mathrm{e}^{\epsilon}\right) \sum_{r=0}^{s} \sum_{m=0}^{\left[\frac{r}{2}\right]} c_{2 m}^{r} t_{r}^{\mu \nu} \sum_{k=1}^{\infty} k^{r+1-2 m} \mathrm{e}^{-k \epsilon} \frac{\partial \ln Z_{s}}{\partial u_{k}^{\mu \nu}}
\end{aligned}
$$

where $c_{m}^{r}=\frac{r !}{m !(r-m) !}$ and $\frac{\partial}{\partial t_{r}} \ln Z$ is the (integrated) bare Green's function given by (2.7). As $\epsilon \rightarrow 0$, divergences appear in $\sum_{k=1}^{\infty}$ in the second line. They also appear in $\frac{\partial}{\partial t_{r}} \ln Z$ for $r=s, s-1$, and in $a$, but those can all be attributed to the counter-term $\Delta a$ when expressed in terms of the renormalized Green's function:

$$
\frac{\partial}{\partial t_{r}} \ln Z_{s}^{\prime}=\frac{\partial}{\partial t_{r}} \ln Z_{s}-\frac{\partial}{\partial t_{r}} \Delta a
$$

For the purpose of demonstrating the cancellation of divergences, it is sufficient to use the minimal form of the counter-term $\Delta a\left(t_{s-1}, t_{s}\right)$ given by $(2.10)$. To isolate various divergences in (3.14), one uses following result:

$$
\lim _{\epsilon \rightarrow 0} \epsilon^{p} \cdot \sum_{k=1}^{\infty} \frac{k^{s+p-3} \mathrm{e}^{-k \epsilon}}{1+t_{s} k^{s-1} \mathrm{e}^{-k \epsilon}}=0, \quad p=1,2,3, \ldots
$$

Using (2.4), (3.15) and (2.10), and dropping terms that vanish as $\epsilon \rightarrow 0$, one finds:

$$
\begin{aligned}
\frac{S_{s}}{Z_{s}} & =\left(\sum_{r=1}^{s} \sum_{m=0}^{\left[\frac{r-1}{2}\right]} c_{2 m+1}^{r} t_{r}^{\mu \nu} \frac{\partial \ln Z_{s}^{\prime}}{\partial t_{r-2 m}^{\mu \nu}}-\sum_{r=0}^{s} t_{r}^{\mu \nu} \frac{\partial \ln Z_{s}^{\prime}}{\partial t_{r}^{\mu \nu}}+a^{\prime}+1\right) \\
& +\epsilon \cdot \operatorname{Tr} \sum_{k=1}^{\infty} \frac{t_{s} k^{s} \mathrm{e}^{-k \epsilon}}{1+t_{s} k^{s-1} \mathrm{e}^{-k \epsilon}} \\
& +\left((s-1) t_{s}^{\mu \nu} \frac{\partial \Delta a}{\partial t_{s}^{\mu \nu}}+(s-2) t_{s-1}^{\mu \nu} \frac{\partial \Delta a}{\partial t_{s-1}^{\mu \nu}}+\Delta a\right)
\end{aligned}
$$

The first term in (3.17) is finite, since it involves renormalized quantities. The second term in (3.17), coming from the second line in (3.14), would not be present 
if the action $S$ was constructed directly in terms of local couplings without the cut-off. This term has a non-trivial contribution even as $\epsilon \rightarrow 0$ because the infinite sum $\sum_{k=1}^{\infty}$ diverges as $\epsilon^{-2}$. The third term is also divergent, and is a function of $t_{s-1}$ and $t_{s}$ only for minimal counter-term $\Delta a$ given by (2.10). Using (2.10) one finds that the $t_{s-1}$-dependent term is in fact finite (for $s>1$ ) in the limit $\epsilon \rightarrow 0$, and is given by:

$$
f_{1}\left(t_{s-1}, t_{s}\right)=(s-1) \operatorname{Tr} \sum_{k=1}^{\infty} \frac{t_{s-1} k^{s-2}}{\left(1+t_{s} k^{s-1}\right)^{2}}
$$

The rest of the third term in (3.17) is a function of $t_{s}$ and is divergent as $\epsilon \rightarrow 0$. But its divergent part cancels precisely that of the second term in (3.17). This remarkable cancellation can be summarized by the following equation:

$$
\begin{aligned}
\epsilon \cdot \operatorname{Tr} \sum_{k=1}^{\infty} \frac{t_{s} k^{s} \mathrm{e}^{-k \epsilon}}{1+t_{s} k^{s-1} \mathrm{e}^{-k \epsilon}}-\operatorname{Tr} \sum_{k=1}^{\infty} \ln \left(1+t_{s} k^{s-1} \mathrm{e}^{-k \epsilon}\right) \\
\quad-(s-1) \operatorname{Tr} \sum_{k=1}^{\infty} \frac{t_{s} k^{s-1} \mathrm{e}^{-k \epsilon}}{1+t_{s} k^{s-1} \mathrm{e}^{-k \epsilon}} \\
=\operatorname{Tr} \sum_{m=1}^{\infty} \frac{1}{m} B_{m(s-1)}\left(t_{s}\right)^{m}+O(\epsilon) \equiv f_{2}\left(t_{s}\right)+O(\epsilon)
\end{aligned}
$$

where $B_{n}$ are the Bernoulli numbers. The finiteness of (3.19) as $\epsilon \rightarrow 0$ can be seen easily by replacing $\sum_{k=1}^{\infty}$ with $\int_{1}^{\infty} \mathrm{d} k$ and integrating by parts. To determine the finite part $f_{2}$, we expand each term in (3.19) in power series of $t_{s}$ (which is absolutely convergent for $\left.t_{s}<(\mathrm{e} \cdot \epsilon)^{s-1} /(s-1)^{s-1}\right)$, and perform the sum $\sum_{k=1}^{\infty}$. The result is a polynomial in $\epsilon$ with $f_{2}$ as the $\epsilon^{0}$ term (the negative powers of $\epsilon$ cancel among the three terms in (3.19)). The cancellation of divergences for $s>1$ means that the string field action $S$ has a well-defined $\epsilon \rightarrow 0$ limit given by:

$S_{s}=\left(\sum_{r=1}^{s} \sum_{m=0}^{\left[\frac{r-1}{2}\right]} c_{2 m+1}^{r} t_{r}^{\mu \nu} \frac{\partial}{\partial t_{r-2 m}^{\mu \nu}}-\sum_{r=0}^{s} t_{r}^{\mu \nu} \frac{\partial}{\partial t_{r}^{\mu \nu}}+f_{1}+f_{2}+a^{\prime}+1\right) Z_{s}^{\prime}\left(t_{r}, a^{\prime}\right), \quad s>1$

where $Z_{s}^{\prime}\left(t_{r}, a^{\prime}\right) \equiv Z_{s}\left(t_{r}, a^{\prime}+\Delta a\right)$ is the renormalized partition function (2.9). 
The analysis of (3.17) for $s=0,1$ is slightly different. For $s=0$, the third term in (3.17) is zero, since $\Delta a$ given in (2.11) is linear in $t_{0}$. The second term in (3.17) is in fact finite as $\epsilon \rightarrow 0$ and gives $\operatorname{Tr}\left(t_{0}\right)$. One obtains the action for the quadratic tachyon:

$$
S_{0}=\left(\operatorname{Tr}\left(t_{0}\right)-t_{0}^{\mu \nu} \frac{\partial}{\partial t_{0}^{\mu \nu}}+a^{\prime}+1\right) Z_{0}^{\prime}, \quad s=0
$$

This agrees completely with the result of [2]. In [2] there is no boundary cutoff and the first term in (3.21) can be traced back to the double contraction in computing the BRST transformation $\{Q, O\}$ of the boundary operator. In the present formulation, there is no double contraction in $\{Q, O\}$ but the first term in (3.21) does appear correctly after the cut-off is removed. Therefore the agreement of (3.21) with [2] is highly non-trivial and provides an important consistency check. For $s=1$ one finds, using (2.12), that divergences cancel between the second and third terms in (3.17) also. But there are now finite contributions from both terms as $\epsilon \rightarrow 0$. Together they give $\operatorname{Tr} t_{0}\left(1-t_{1}^{2}\right)^{-1}$, and the action is,

$$
S_{1}=\left(\operatorname{Tr} \frac{t_{0}}{1-t_{1}^{2}}-t_{0}^{\mu \nu} \frac{\partial}{\partial t_{0}^{\mu \nu}}+a^{\prime}+1\right) Z_{1}^{\prime}, \quad s=1
$$

From the computations described above, it appears miraculous that the counter-term $\Delta a$ that makes the matter partition function finite in Section 2 cancels all divergences in the action (3.14). To see that this cancellation is non-trivial, we first note that the action is determined from the bare two-point correlation functions of the world-sheet theory; there is no counter-term or renormalization other than those present in the world-sheet Lagrangian. When expressed in terms of renormalized couplings, the action (3.14) contains divergences (the third term in (3.17)) coming from the world-sheet counter-term $\Delta a$. The additional divergence (the second term in (3.14) or (3.17)) can be traced back to the BRST transformation of the leading unrenormalizable boundary operator $X \partial_{\theta}^{s} X$. Because $\left\{Q, X_{\mu}(\theta)\right\}=c \partial_{\theta} X_{\mu}(\theta)$, the operator $X \partial_{\theta}^{s+1} X$ with one higher dimension may 
be produced by BRST transformation, and its two-point function will contribute to the string field action. More precisely, as can be seen from the second term in $A(\theta)$, this contribution is multiplied by the short-distance cut-off $\left(\sin \left(\theta-\theta^{\prime}\right) \sim \epsilon\right.$ ) and it corresponds to precisely the divergent second term in (3.14) and (3.17). From the world-sheet point of view, correlation functions of boundary operators $X \partial_{\theta}^{s+1} X$ can be made finite only if the correponding coupling and its renormalization is introduced. Then by induction one would seem to require an infinite number of couplings and their renormalization to obtain a finite string field action as $\epsilon \rightarrow 0$. What actually happens is a complete concellation of divergence with only renormalization of a finite number of local couplings.

Other choices of counter-term $\Delta a$ with different finite part will also give a finite and cut-off independent string field action (3.20), but with possibly different finite term $f_{1}+f_{2}$. For the minimal counter-term, the action (3.20) is not $s$-independent, as the $t_{s} \rightarrow 0$ limit is singular. As shown in Section two, a non-minimal but $s$ independent counter-term can been constructed to give a $s$-independent renormalized partition function. Unfortunately these $s$-independent counter-terms still do not give an $s$-independent action. This is because of the explicit $s$-dependence of the second term in (3.17); the two limits $\epsilon \rightarrow 0$ and $t_{s} \rightarrow 0$ do not commute for this term. The failure of the action to be $s$-independent may present difficulties to the notion that the space of open string theories should be represented by local world-sheet boundary interactions. It could be that, in fact (as has been suggested to us by D. Gross), the cut-off should only be removed if one is sufficiently close to classical solutions. (For the specific boundary interactions considered in this paper, the massive space-time fields are taken at zero momentum and so are far off-shell.) However, we do not know any systematic way to proceed - or to achieve background independence - along those lines. We do believe that the problem of interpreting or dealing with the unrenormalizable world-sheet interactions is one of the main obstructions to progress in string theory.

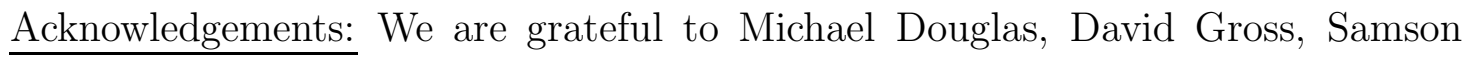


Shatashvili and Erik Verlinde for helpful discussions.

\section{REFERENCES}

1. E. Witten, On Background Independent Open-String Field Theory, Phys. Rev. D46 (1992) 5467.

2. E. Witten, Some Computations in Background Independent Off-Shell String Theory, IASSNS/HEP-92/63.

3. E. Fradkin and A. A. Tseytlin, Non-Linear Electrodynamics from Quantized Strings,, Phys. Lett. B163 (1985) 123.

4. A. Abouelsaood, C. G. Callan, C. R. Nappi, and S. A. Yost, Open Strings in Background Gauge Fields, Nucl. Phys. B280 (1987) 599.

5. S. Shatashvili and E. Verlinde, private communications 